\title{
Pyoderma gangrenosum: a rare disease with a rare localization
}

\author{
Ionela-Alina Grosu1, Delia Gabriela Ciobanu-Apostol, ${ }^{1,2}$, Dan Vâță ${ }^{1,3}$, Elena \\ Andrese $^{1,3}$, Laura Gheucă-Solovăstru*,1,3, Laura Stătescu, ${ }^{1,3}$
}

1"Grigore T. Popa" University of Medicine and Pharmacy lasi, Romania, ${ }^{2}$ Department of Pathology, "Sf. Spiridon" Clinical Emergency Hospital, Iasi, Romania, ${ }^{3}$ Department of Dermatology, "Sf. Spiridon" Hospital, lasi, Romania

\begin{abstract}
Pyoderma gangrenosum (PG) is a rare neutrophilic inflammatory disease, characterized by necrotizing and painful ulcers of unknown cause, that it is often misinterpreted. PG is usually idiopathic and includes dysregulation of the autoimmune system and frequently has an association with other systemic diseases (inflammatory bowel disease, rheumatological, hematological disease or malignancy). The most common localization is on the lower legs, mainly in the pretibial area. We describe a case of Pyoderma gangrenosum in a 43 year-old female patient with erythemato-edematous big plaque, covered with sphacelous, yellow-green pus, localized on the left inguinal area and pubic area, which is a rare localization of this disease.
\end{abstract}

Keywords: pyoderma gangrenosum, idiopathic disease, ulceration, pubic area

\section{Introduction}

Pyoderma gangrenosum (PG) is an uncommon neutrophilic inflammatory disease, with low incidence in medical practice and usually idiopathic. This dermatosis is characterized by an infiltration with neutrophils in the dermis and secondary tissue destruction [1]. It was first described by Louis Brocq in 1916 as "phagédénisme géométrique", to highlight the geometrical pattern of the ridge and the rapidly extensive ulcerative necrotic lesion [2], and later the term PG was introduced in 1930 by Brunsting et al [3]. The incidence of $P G$ is estimated to be 3 to 10 patients per million population per year [4]

Received: November 2016; Accepted after review: November 2016; Published: December 2016.

${ }^{*}$ Corresponding author: Prof. Laura GheucăSolovăstru, MD, PhD, Department of Dermatology, "Sf. Spiridon" Hospital, lasi, "Grigore T. Popa" University of Medicine and Pharmacy Iasi, 16 Universitatii Street, 700115, lasi, Romania.

E-mail: Isolovastru13@yahoo.com
The peak incidence occurs between the second and fifth decade of life with female preponderance. The most frequent occurrences of PG happen in adults, but on rare occasions it can also affect children [5]. The extension of the initial lesion determined by repetitive trauma in patients with $P G$ is defined as pathergy phenomenon. The most common localization is on the lower legs, mainly in the pretibial area. It can also occur on other sites of the body such as breasts, hands, trunk, head and neck, and peristomal skin [5]. Five clinical variants are currently recognized: classic (ulcerative), bullous, pustular, vegetative, and peristomal types [6]. PG presents the pathergy phenomenon in approximately $20 \%$ of cases, in which injury of the skin often initiates a new lesion or intensifies a preexisting one [7].

In $50 \%$ to $70 \%$ of cases are associated with a variety of systemic pathology that can occur before, concomitantly, or after the diagnosis of PG. The most common associated diseases are solid organ 
malignancies [7], inflammatory bowel disease (ulcerative colitis, diverticulosis, Crohn's disease) [8], seropositive or seronegative arthritis [9], paraproteinemia (particularly $\lg \mathrm{A}$ ) [9], and hematological diseases (myelogenous leukemia, hairy cell leukemia, myelofibrosis, and monoclonal gammopathy) [10]. PG has been reported in association with other neutrophilic dermatosis, such as Behcet's disease, subcorneal pustular dermatosis, Sweet's syndrome (acute febrile neutrophilic dermatosis), and erythema elevatum diutinum [10].

The etiology of $P G$ is unknown and the pathophysiology poorly understood [1, 10], but the main hypothesis is that of a neutrophilic dysfunction which is suggested by the aberrant neutrophil chemotaxis and inconsistencies in phagocytosis. The overexpression of IL-8, IL-17, tumor necrosis factor-alfa, chemokines $1,2,3$, and 16, and matrix metalloproteinase 2 and 9 [10], suggests that $P G$ represents a hyperreactive response to inflammatory, neoplastic or traumatic process in susceptible persons.

Pain is the major symptom responsible for reduction in the quality of life and morbidity [10]. Skin biopsy samples taken from the necrotic, undermined ulcer border of PG reveal mixed cellular inflammation with the predominance of neutrophils [11]. Clinically, the lesion typically starts as a tender nodule, plaque or sterile pustule that enlarges and erodes, over a course of days, into a sharply edged ulcer with undermined, violaceous borders and erythema [11]. PG continues to be a diagnosis of exclusion [11], which can often lead to misdiagnosis.

The variousness of systemic therapy used to treat pyoderma gangrenosum shows the difficulty of managing this disorder and the lack of uniform responses to treatment by the patients. Systemic steroids were by far the most commonly prescribed treatment [12].

The long-term prognosis of $P G$ for most patients is good, especially in those who have no other underlying associated disease [13].

\section{Case report}

We hereby present the case of a 43 yearold Caucasian female patient with an inflammatory painful nodule, about $0.5 / 1 \mathrm{~cm}$ diameter, which expressed a purulent discharge at pressure, observed by the patient three weeks before admission in Dermatology Clinic, "Sf. Spiridon" Hospital, lasi.

Repeated trauma to the lesion led to its extension, to the occurrence of an erythematous and edematous big plaque with pustules on the surface, intensely painful, so the patient went to a surgical department in the territory.

A surgery consultation was initiated for debridement of necrotic wound areas. After extensive surgical debridement, the wound permanently enlarge, so the diagnosis of pyoderma gangrenosum was suspected. Cultures were sterile for bacteria and fungi.

The patient was admitted to our Dermatology Clinic where the skin examination revealed erythemato-edematous big plaque, uneven margins, $20 / 15 \mathrm{~cm}$ in diameter, $2 \mathrm{~cm}$ depth, covered with sphacelous, debris, yellow-green pus, localized on the left inguinal area and pubic area (Figure 1). The only symptom described by the patient was pain.

Paraclinical investigations (complete blood count, inflammatory markers, biochemical investigations, serum glucose, liver and renal function tests) were in normal parameters. Bacterial and fungal cultures were negative for infection. Clinical and laboratory investigations revealed no associated pathologies.

Pathologic examination of the punch biopsy taken from right ulcer border revealed ulceration in the thickness of dermis (Figure 2), vascular dilatation and perivascular lymphocytes (Figure 3), and massive polymorphous dermal inflammatory infiltrate (Figure 4). 


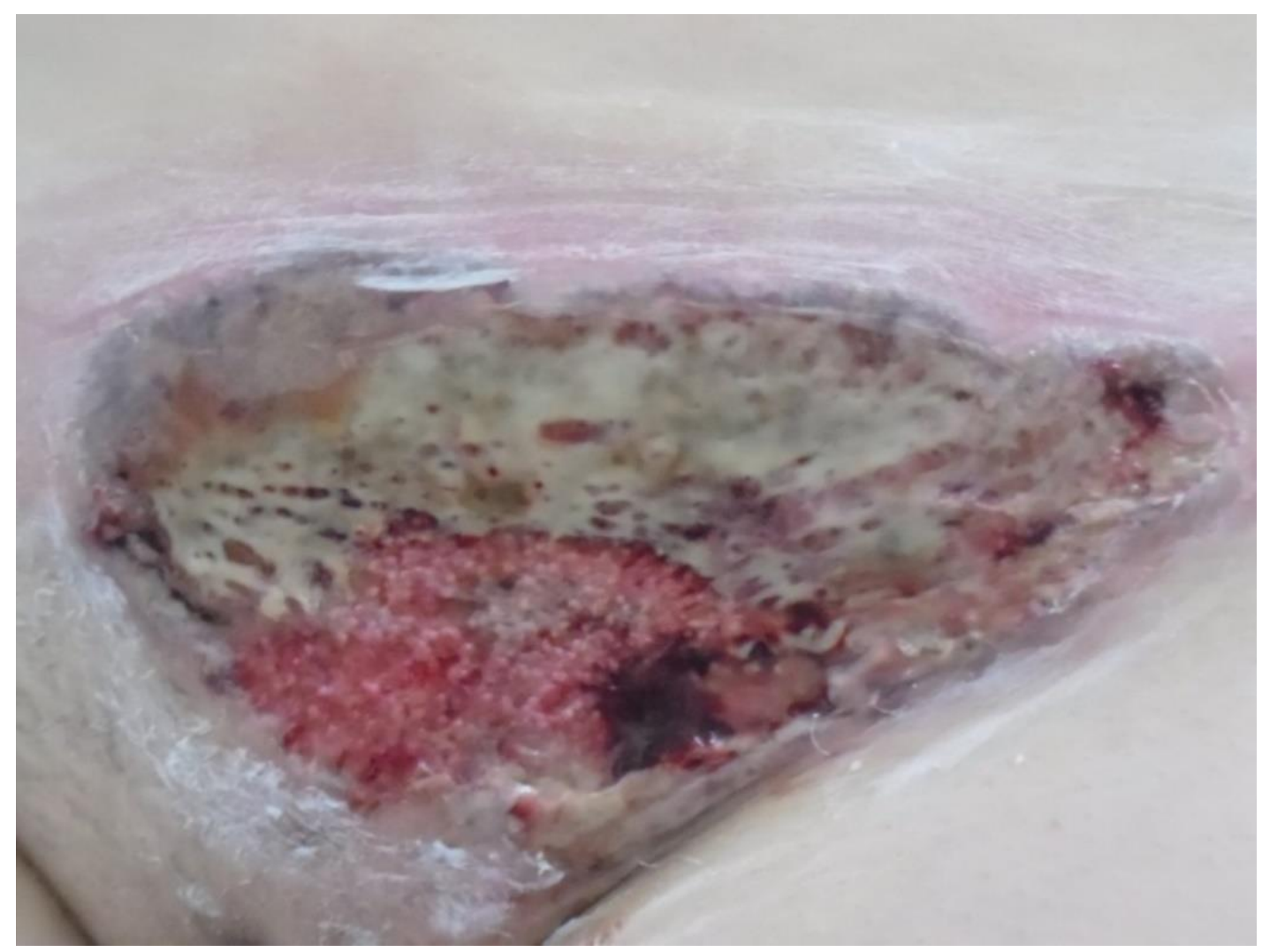

Fig. 1. Clinical aspect of the lesion at admission in Dermatology Clinic, localized on the left inguinal area and pubic area

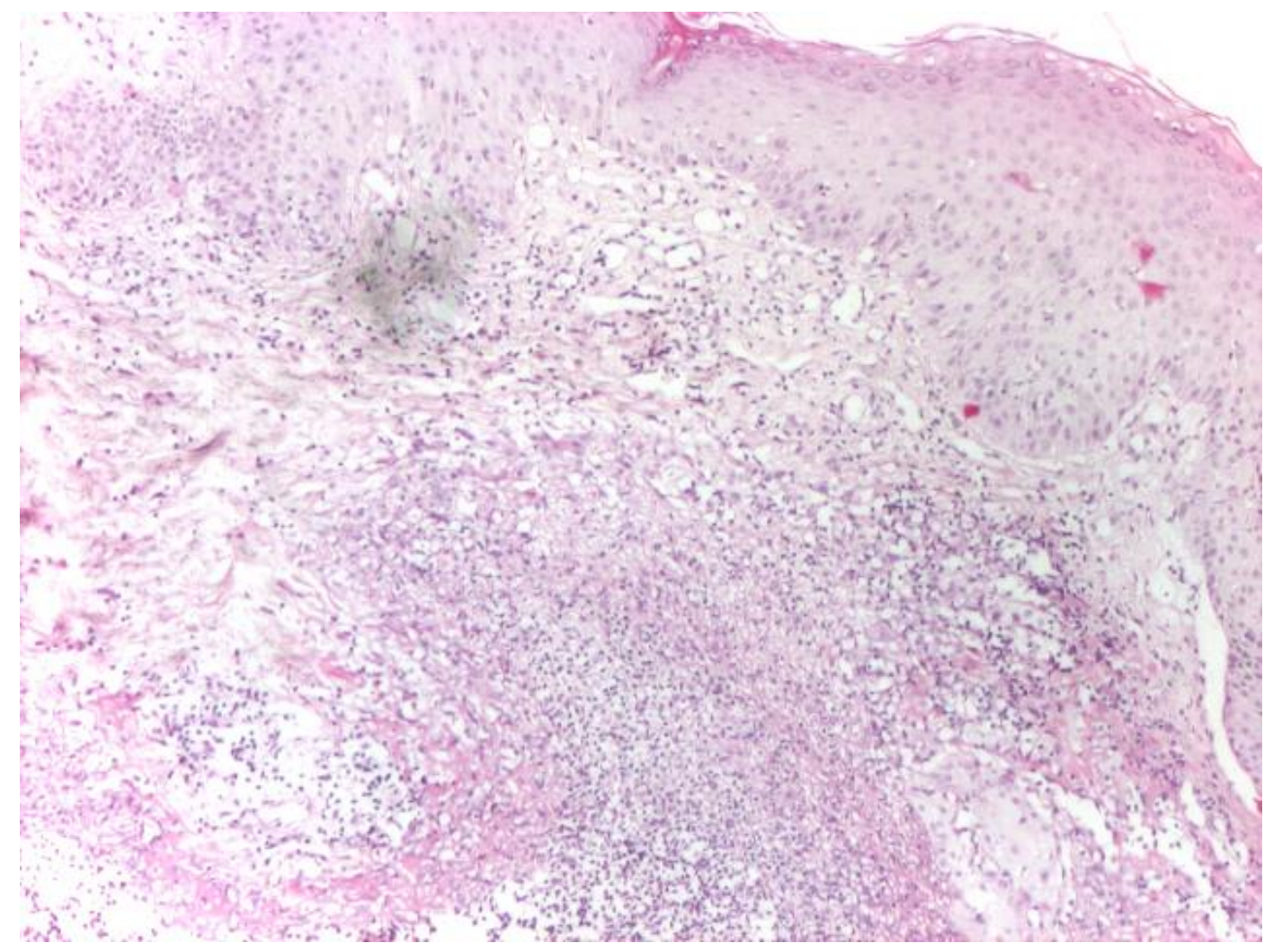

Fig. 2. Biopsy showing ulceration in the thickness of dermis (HE, $x 40)$ 


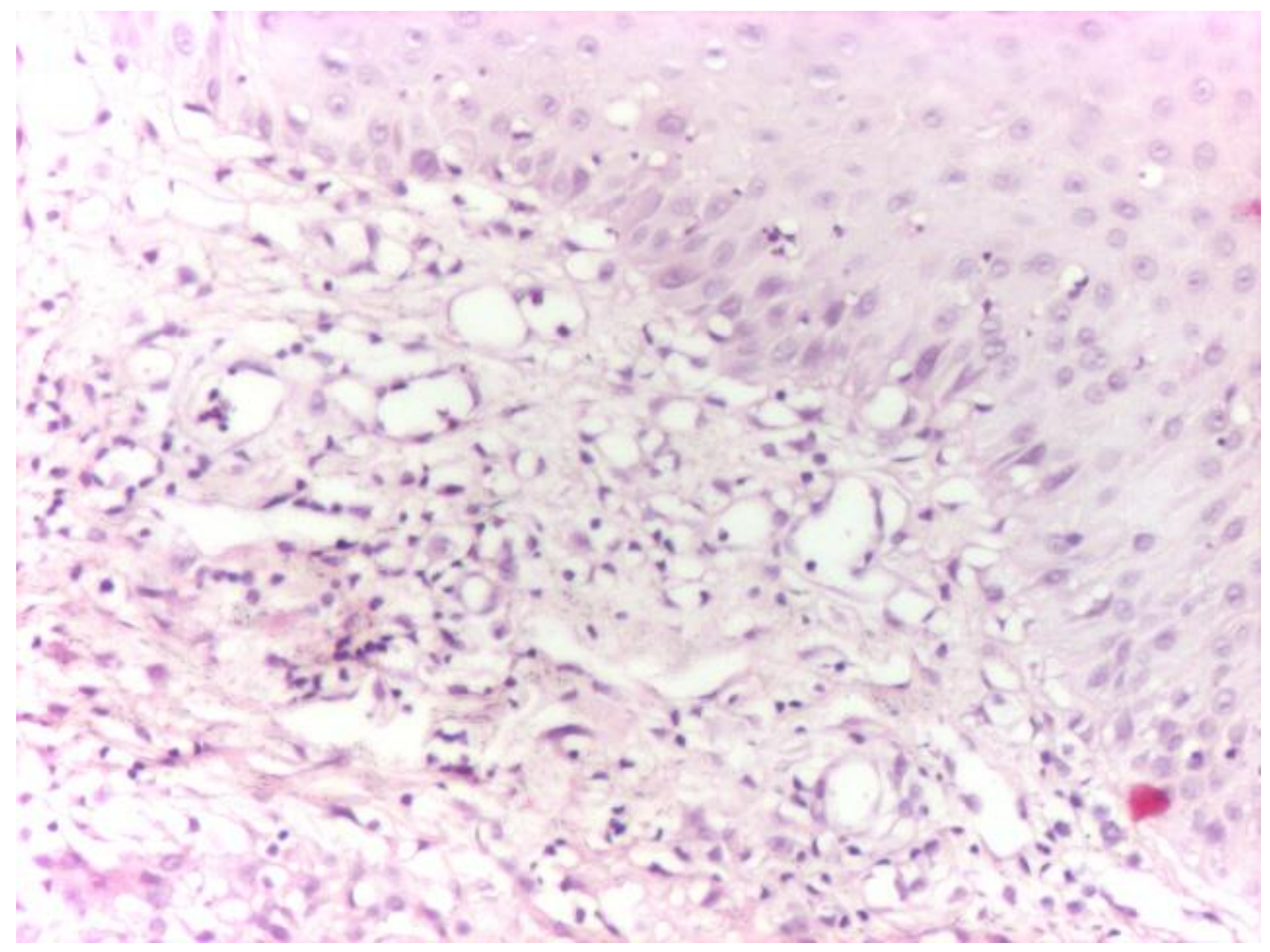

Fig. 3. Histopathologic image of the lesion showing vascular dilatation and perivascular lymphocytes (HE, x100)

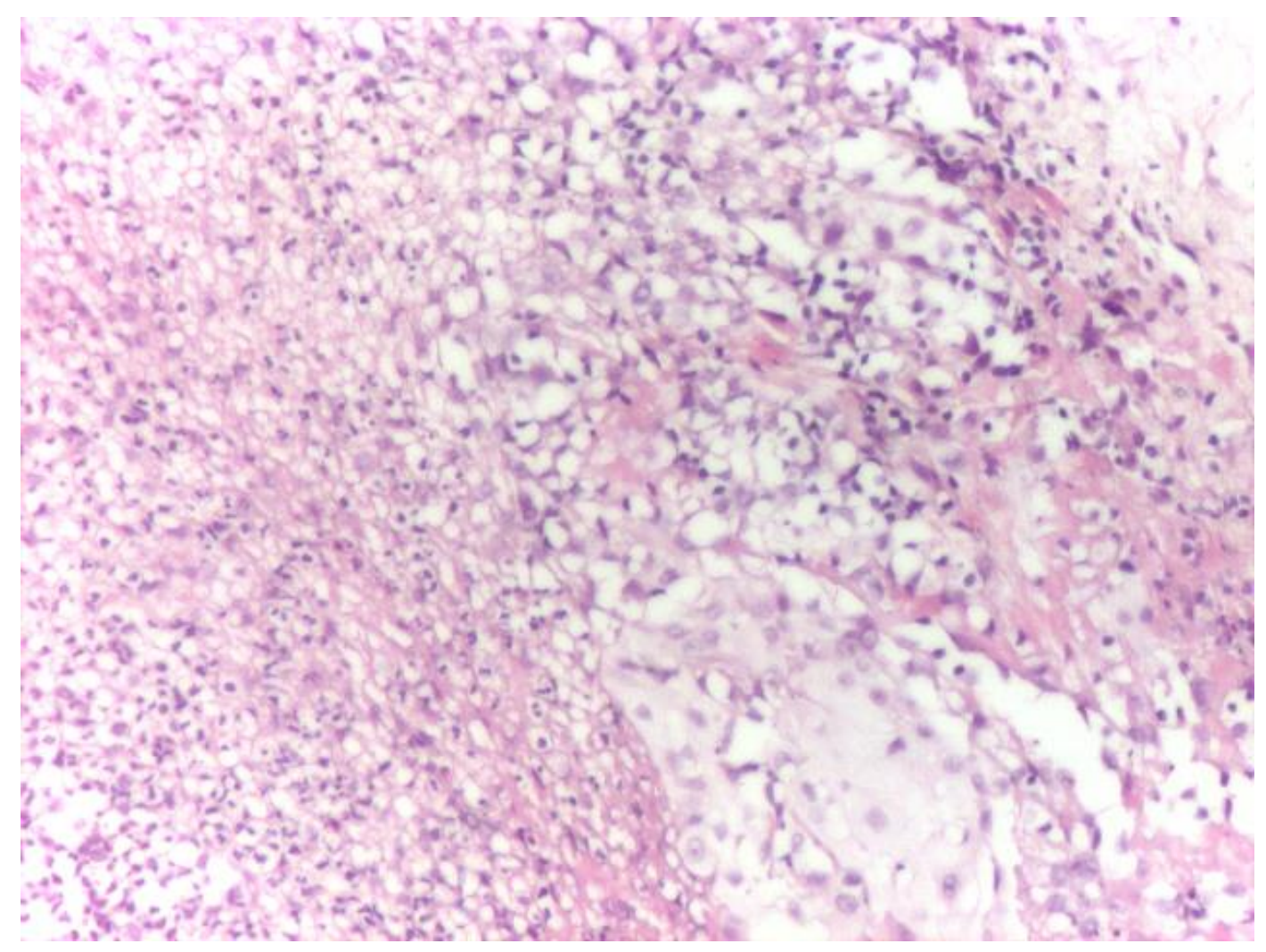

Fig. 4. Histopathology of the skin lesion. Lesion showing massive polymorphous dermal inflammatory infiltrate (HE, x100) 
The clinical course of the lesion, its morphology, and the histopathological findings suggested a diagnosis of PG. This rare neutrophilic dermatosis was confirmed and the patient was commenced on concomitant therapy with pulsed intravenous dexamethasone (2 vials per day, 1 vial $=2$ $\mathrm{mL}=8 \mathrm{mg}$ dexamethasone sodium phosphate), oral prednisone (1 $\mathrm{mg} / \mathrm{kg}$ per day), topical fusidic acid and betamethasone and systemic antibiotherapy (intravenous colistine), resulting in rapid improvement in skin conditions (Figure $5)$.

We chose to administrate a combination of intravenous dexamethasone and oral prednisone due to the severity of the lesion, and colistine for its role in the prevention of nosocomial infection due to repeated surgical debridement and the immunosuppression induced by the corticosteroid treatment.

The edema and induration surrounding the lesion had disappeared and the ulcer had begun to close. The lesion was rapidly healing, with a cribriform atrophic scar, after one month of treatment (Figure 6).

In evolution, the patient had a relapse, 6 months later. The new lesion was located in the right armpit. This time, the patient was quickly diagnosed, and the skin lesions had a favorable response receiving concomitant therapy with pulsed intravenous dexamethasone (2 vials per day, 1 vial $=2$ $\mathrm{mL}=8 \mathrm{mg}$ dexamethasone sodium phosphate), oral prednisone (1 $\mathrm{mg} / \mathrm{kg}$ per day), topical fusidic acid and betamethasone and systemic antibiotherapy (intravenous colistine).

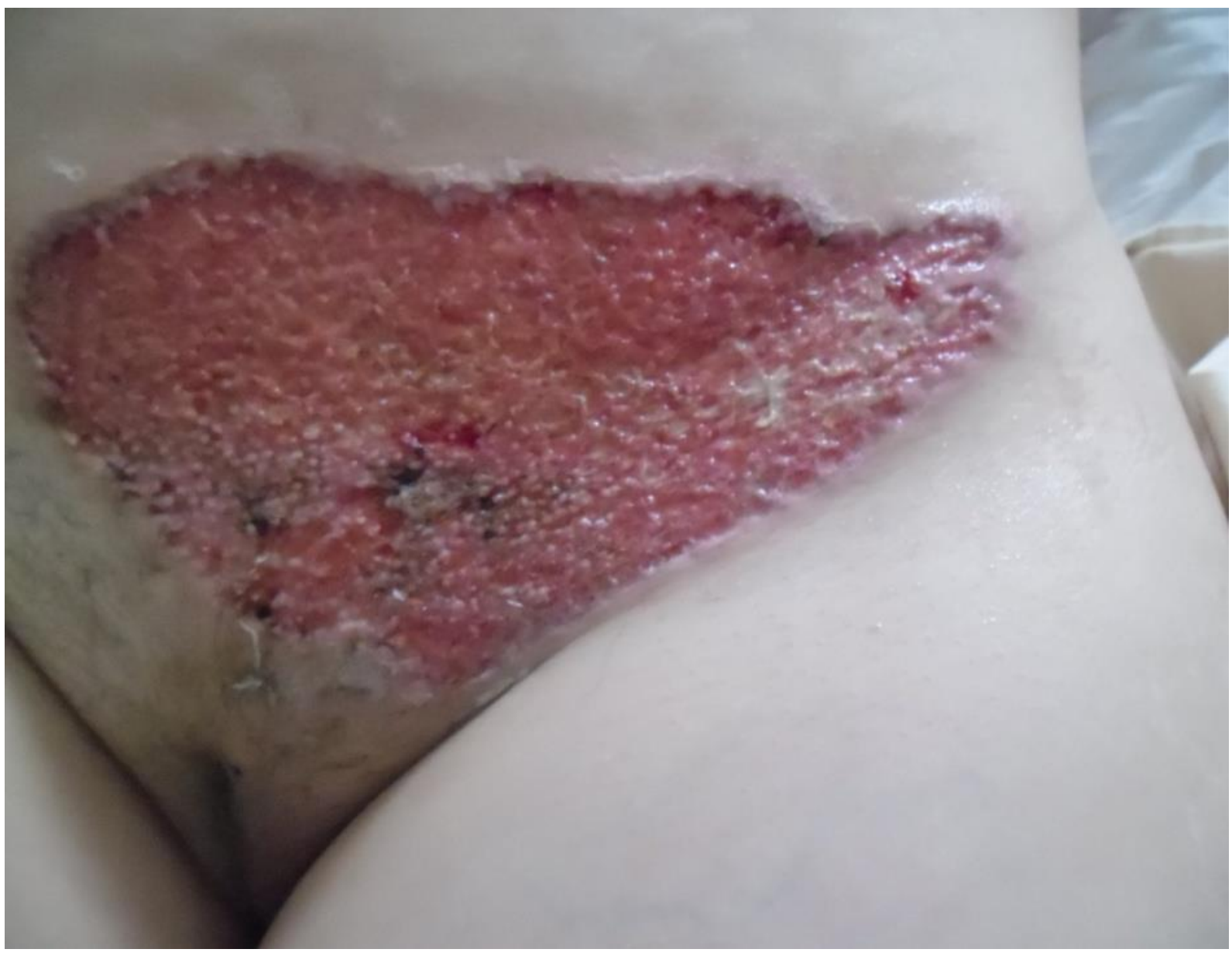

Fig. 5. The clinical aspect of the lesion, two weeks later 


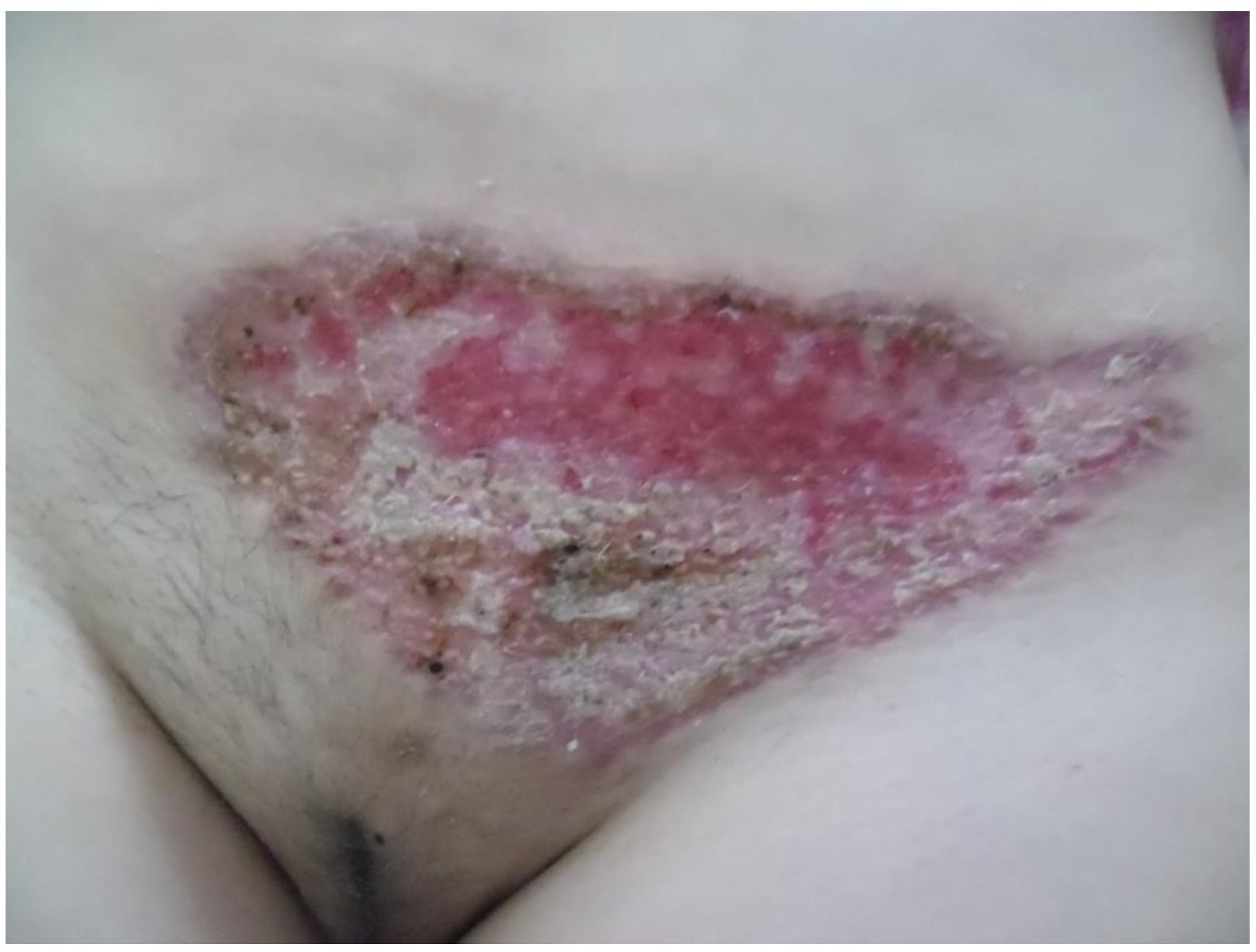

Fig. 6. Rapid evolution, to the healing of the ulceration, with re-epithelization and scarring, one month later

\section{Discussions}

Pyoderma gangrenosum $(P G)$ is a rare skin disorder that may arise spontaneously, or following repeated trauma on the lesion or cutaneous injury. The underlying cause of pathergy is considered to be the increased activity of polymorphonuclear leukocytes.

Differential diagnosis of $P G$ includes infections (abscess) [14].

Despite the significant advances in the therapeutic management, pyoderma gangrenosum remains a potentially lethal disease with elevated levels of morbidity and mortality (the risk of death was shown to be three times higher than the general population). Due to this, long-term and regular follow-ups are requested of these patients for the rest of their life in order to evaluate the development of myeloproliferative disorders [15].
The social and personal-life of patients who are diagnosed with $P G$ are mainly influenced in a negative way which is why a multidisciplinary care is required.

The particularities of our case report consist in the rarity of this disease, atypical localization (pubic area), idiopathic lesion and the delay of the diagnosis.

\section{Conclusions}

Pyoderma gangrenosum is a disease with an incompletely elucidated etiology, underdiagnosed, which requires anatomical and pathological confirmation and must be taken into discussion in cases of ulceration with sudden onset, without clues of possible etiology.

PG's diagnosis is difficult, particularly for doctors from other specialties who rarely recognize this entity, but also for 
dermatologists, as there is no pathognomonic sign, clinical or histological feature.

The patient must be followed up, because of the frequent association with important systemic diseases.

This case highlights the importance of a thorough history and systems review of all

\section{References}

1. Conrad C, Trüeb RM. Pyoderma gangrenosum. J Dtsch Dermatol Ges 2005; 3(5):334-342.

2. Brocq L. Nouvelle contribution a l'étude du phagédénisme géométrique. Ann Dermatol Syphiligr 1916; 6:1-39.

3. Brunsting AL, Goeckerman WH, O'Leary PA. Pyoderma gangrenosum: clinical and experimental observation in five cases occuring in adults. Arch Derm Syphilol 1930; 22: 65580.

4. Braswell SF, Kostopoulos TC, Ortega-Loayza AG. Pathophysiology of pyoderma gangrenosum (PG): an updated review. J Am Acad Dermatol 2015; 73(4):691-8. [Article in Press]

5. Pereira N, Brites MM, Gonçalo M, Tellechea O, Figueiredo A. Pyoderma gangrenosum--a review of 24 cases observed over 10 years. Int J Dermatol 2013; 52(8):938-45.

6. Ahronowitz I, Harp J, Shinkai K. Etiology and management of pyoderma gangrenosum: a comprehensive review. Am J Clin Dermatol 2012; 13(3):191-211.

7. Cabalag MS, Wasiak J, Lim SW, Raiola FB. Inpatient management of pyoderma gangrenosum: treatments, outcomes, and clinical implications. Ann Plast Surg 2015; 74(3):354-60.

8. Al Ghazal $\mathrm{P}$, Herberger $\mathrm{K}$, Schaller J, et al. Associated factors and comorbidities in patients with pyoderma gangrenosum in patients and underlines the role of multidisciplinary care.

\section{Conflict of interest}

Authors declare no conflicts of interest.
Germany: a retrospective multicentric analysis in 259 patients. Orphanet J Rare Dis 2013; 8:136.

9. Su WP, Davis MD, Weenig RH, Powell FC, Perry HO. Pyoderma gangrenosum: clinicopathologic correlation and proposed diagnostic criteria. Int J Dermatol 2004; 43(11):790-800.

10. Adışen E, Erduran F, Gürer MA. Pyoderma Gangrenosum: A Report of 27 Patients. Int J Low Extrem Wounds 2016; 15(2):148-54.

11. Al Ghazal P, Dissemond J. Therapy of pyoderma gangrenosum in Germany: results of a survey among wound experts. J Dtsch Dermatol Ges 2015; 13(4):317-24.

12. Binus AM, Qureshi AA, Li VW, Winterfield LS. Pyoderma gangrenosum: a retrospective review of patient characteristics, comorbidities and therapy in 103 patients. $\mathrm{Br} J$ Dermatol 2011; 165(6):1244-50.

13. Callen JP, Jackson JM. Pyoderma gangrenosum: an update. Rheum Dis Clin North Am 2007; 33(4):787-802, vi.

14. Dabade TS, Davis MD. Diagnosis and treatment of the neutrophilic dermatoses (pyoderma gangrenosum, Sweet's syndrome). Dermatol Ther 2011; 24(2):273-84.

15. Gameiro A, Pereira N, Cardoso JC, Gonçalo M. Pyoderma gangrenosum: challenges and solutions. Clin Cosmet Investig Dermatol 2015; 8:285-93. 